IJMMS 27:9 (2001) 535-539

PII. S016117120100713X

http://ijmms.hindawi.com

(c) Hindawi Publishing Corp.

\title{
ON GENERALIZATIONS OF REGULAR-LINDELÖF SPACES
}

\author{
ANWAR JABOR FAWAKHREH and ADEM KILIÇMAN
}

(Received 10 November 2000 and in revised form 28 February 2001)

\begin{abstract}
We study nearly regular-Lindelöf, almost regular-Lindelöf and weakly regularLindelöf spaces. Characterizations and some properties for these spaces are proposed. Relations among them are also studied.
\end{abstract}

2000 Mathematics Subject Classification. 54A05, 54D15, 54D20, 54G05.

1. Introduction. In 1959 Frolik [4] introduced weakly Lindelöf space that afterward was studied by several authors. In 1982 Balasubramanian [1] introduced and studied nearly Lindelöf spaces. In 1984 Willard and Dissanayake [8] introduced and studied almost Lindelöf spaces and in 1996 Cammaroto and Santoro [3] introduced nearly regular-Lindelöf, almost regular-Lindelöf and weakly regular-Lindelöf spaces on using regular covers.

In this paper, a space $X$ means a topological space $(X, \tau)$ on which no separation axioms are assumed unless explicitly stated otherwise. The interior and the closure of any subset $A$ of $X$ will be denoted by $\operatorname{Int}(A)$ and $\mathrm{Cl}(A)$, respectively. By regularly open cover of $X$ we mean a cover of $X$ by regularly open sets in $(X, \tau)$.

Recall that a subset $A \subseteq X$ is called regularly open (regularly closed) if $A=\operatorname{Int}(\mathrm{Cl}(A))$ $(A=\mathrm{Cl}(\operatorname{Int}(A)))$. The generated space by the regularly open subsets of $(X, \tau)$ is denoted by $\left(X, \tau^{*}\right)$ and is called the semiregularization of $X$. If $\tau=\tau^{*}$ then $X$ is said to be semiregular. A space $X$ is almost regular if and only if for any regularly closed set $C$ and any singleton $\{x\}$ disjoint from $C$ there exist two disjoint open sets $U$ and $V$ such that $C \subseteq U$ and $x \in V$. Note that a space $X$ is regular if and only if it is semiregular and almost regular (see [5]). Moreover, a space $X$ is called extremally disconnected if and only if the closure of every open set in $X$ is open. A space $X$ is called nearly paracompact (see [6]) if and only if every regularly open cover of $X$ admits an open locally finite refinement.

DefinItion 1.1 (see $[1,4,8]$ ). A topological space $X$ is said to be nearly Lindelöf, almost Lindelöf, and weakly Lindelöf if, for every open cover $\left\{U_{\alpha}: \alpha \in \Delta\right\}$ of $X$, there exists a countable subset $\left\{\alpha_{n}: n \in \mathbb{N}\right\} \subseteq \Delta$ such that

$$
X=\bigcup_{n \in \mathbb{N}} \operatorname{Int}\left(\mathrm{Cl}\left(U_{\alpha_{n}}\right)\right), \quad X=\bigcup_{n \in \mathbb{N}} \mathrm{Cl}\left(U_{\alpha_{n}}\right), \quad X=\mathrm{Cl}\left(\bigcup_{n \in \mathbb{N}} U_{\alpha_{n}}\right),
$$

respectively. 
Note that a nearly Lindelöf space is almost Lindelöf and an almost Lindelöf space is weakly Lindelöf, but none of the two converses is true. Also it is clear that separable spaces are weakly Lindelöf. Moreover, a semiregular and nearly Lindelöf space is Lindelöf [3].

DEFINITION 1.2 (see [2]). An open cover $\left\{U_{\alpha}: \alpha \in \Delta\right\}$ of a topological space $X$ is called regular cover if, for every $\alpha \in \Delta$, there exists a nonempty regularly closed subset $C_{\alpha}$ of $X$ such that $C_{\alpha} \subseteq U_{\alpha}$ and $X=\bigcup_{\alpha \in \Delta} \operatorname{Int}\left(C_{\alpha}\right)$.

\section{Almost regular-Lindelöf spaces}

DEFINITION 2.1 (see [3]). A topological space $X$ is called almost regular-Lindelöf if every regular cover $\left\{U_{\alpha}: \alpha \in \Delta\right\}$ of $X$ admits a countable subfamily $\left\{U_{\alpha_{n}}: n \in \mathbb{N}\right\}$ such that $X=\bigcup_{n \in \mathbb{N}} \mathrm{Cl}\left(U_{\alpha_{n}}\right)$.

Obviously, every almost Lindelöf space is almost regular-Lindelöf, but the converse is not true, in general (see [3, Example 3.11 and Property 4.5]). Also weakly Lindelöf spaces need not be almost regular-Lindelöf (see Example 4.5 below).

REMARK 2.2. Every regular cover $\left\{U_{\alpha}: \alpha \in \Delta\right\}$ of $X$ admits a refinement of regularly open sets in $X$ covers $X$. Since if $\left\{U_{\alpha}: \alpha \in \Delta\right\}$ is a regular cover of $X$, then for each $\alpha \in \Delta$ there exists a nonempty regularly closed subset $C_{\alpha}$ with $C_{\alpha} \subseteq U_{\alpha}$ and $X=$ $\bigcup_{\alpha \in \Delta} \operatorname{Int}\left(C_{\alpha}\right)$. So $\left\{\operatorname{Int}\left(C_{\alpha}\right): \alpha \in \Delta\right\}$ is a regularly open cover of $X$ refines $\left\{U_{\alpha}: \alpha \in \Delta\right\}$.

REMARK 2.3. If $(X, \tau)$ is almost regular space then every regularly open cover $\left\{U_{\alpha}\right.$ : $\alpha \in \Delta\}$ of $X$ admits a regular cover $\left\{W_{\gamma}: \gamma \in \Gamma\right\}$ refines $\left\{U_{\alpha}: \alpha \in \Delta\right\}$. Since if $\left\{U_{\alpha}: \alpha \in\right.$ $\Delta\}$ is a regularly open cover of the almost regular space $X$ and $U_{\alpha_{x}}$ containing $x$, there exist two regularly open subsets $V_{\alpha_{x}}$ and $W_{\alpha_{x}}$ such that $x \in V_{\alpha_{x}} \subseteq \mathrm{Cl}\left(V_{\alpha_{x}}\right) \subseteq W_{\alpha_{x}}$ and $\mathrm{Cl}\left(W_{\alpha_{x}}\right) \subseteq U_{\alpha_{x}}$ (see [5, Theorem 2.2]). So the family $\left\{W_{\alpha_{x}}: x \in X\right\}$ is a regular cover of $X$ that refines $\left\{U_{\alpha}: \alpha \in \Delta\right\}$.

Proposition 2.4 (see [3]). An almost regular and almost regular-Lindelöf space $X$ is nearly Lindelöf.

COROLLARY 2.5. A regular and almost regular-Lindelöf space $X$ is Lindelöf.

Proposition 2.6. Let $(X, \tau)$ be an extremally disconnected and almost regularLindelöf space then it is nearly Lindelöf.

Proof. Let $\left\{U_{\alpha}: \alpha \in \Delta\right\}$ be an open cover of $X$. Since $(X, \tau)$ is extremally disconnected, $\left\{\mathrm{Cl}\left(U_{\alpha}\right): \alpha \in \Delta\right\}$ is a regular cover of the almost regular-Lindelöf space $X$. So there exists a countable subset $\left\{\alpha_{n}: n \in \mathbb{N}\right\} \subseteq \Delta$ such that $X=\bigcup_{n \in \mathbb{N}} \operatorname{Cl}\left(U_{\alpha_{n}}\right)=$ $\bigcup_{n \in \mathbb{N}} \operatorname{Int}\left(\mathrm{Cl}\left(U_{\alpha_{n}}\right)\right)$. This implies that $X$ is nearly Lindelöf and completes the proof.

\section{Nearly regular-Lindelöf spaces}

DEFINITION 3.1 (see [3]). A topological space $X$ is called nearly regular-Lindelöf if every regular cover $\left\{U_{\alpha}: \alpha \in \Delta\right\}$ of $X$ admits a countable subfamily $\left\{U_{\alpha_{n}}: n \in \mathbb{N}\right\}$ such that $X=\bigcup_{n \in \mathbb{N}} \operatorname{Int}\left(\mathrm{Cl}\left(U_{\alpha_{n}}\right)\right)$. 
It is clear that every nearly Lindelöf space is nearly regular-Lindelöf but the converse is not true in general. The space $X=(\mathbb{R}, \sigma)$ in [3, Example 3.11] was shown not to be almost Lindelöf so it is not nearly Lindelöf. By noting that $\operatorname{Int}_{\tau_{u}}\left(\mathrm{Cl}_{\sigma}\left(U_{\alpha_{n}}\right)\right) \subseteq$ $\operatorname{Int}_{\sigma}\left(\mathrm{Cl}_{\sigma}\left(U_{\alpha_{n}}\right)\right)$ where $\tau_{u}$ denotes the usual topology on $\mathbb{R}$, we conclude that $X=$ $\bigcup_{n \in \mathbb{N}} \operatorname{Int}_{\sigma}\left(\mathrm{Cl}_{\sigma}\left(U_{\alpha_{n}}\right)\right)$, which implies that $X$ is nearly regular-Lindelöf.

Proposition 3.2. If the space $X$ is almost Lindelöf then it is nearly regular-Lindelöf.

Proof. Let $\left\{U_{\alpha}: \alpha \in \Delta\right\}$ be a regular cover of $X$. By Remark 2.2, there exists a family of regularly closed sets $\left\{C_{\alpha}: \alpha \in \Delta\right\}$ such that $\left\{\operatorname{Int}\left(C_{\alpha}\right): \alpha \in \Delta\right\}$ is a (regularly) open cover of the almost Lindelöf space $X$ that refines $\left\{U_{\alpha}: \alpha \in \Delta\right\}$. So there exists a countable subset $\left\{\alpha_{n}: n \in \mathbb{N}\right\} \subseteq \Delta$ such that $X=\bigcup_{n \in \mathbb{N}} \mathrm{Cl}\left(\operatorname{Int}\left(C_{\alpha_{n}}\right)\right)=\bigcup_{n \in \mathbb{N}} C_{\alpha_{n}} \subseteq$ $\bigcup_{n \in \mathbb{N}} U_{\alpha_{n}} \subseteq \bigcup_{n \in \mathbb{N}} \operatorname{Int}\left(\mathrm{Cl}\left(U_{\alpha_{n}}\right)\right)$. This implies that $X$ is nearly regular-Lindelöf and completes the proof.

Moreover, it is clear that every nearly regular-Lindelöf space is almost regularLindelöf. Weakly Lindelöf spaces need not be nearly regular-Lindelöf as Example 4.5 shows.

Now using Proposition 2.4, it is easy to prove the following proposition.

PROPOSITION 3.3. An almost regular and nearly regular-Lindelöf space $X$ is nearly Lindelöf.

The following proposition gives a characterization of nearly regular-Lindelöf spaces.

Proposition 3.4. A space $X$ is nearly regular-Lindelö if and only if for every family $\left\{C_{\alpha}: \alpha \in \Delta\right\}$ of regularly closed subsets of $X$ such that, for each $\alpha \in \Delta$, there exists an open set $A_{\alpha} \supseteq C_{\alpha}$ with $\bigcap_{\alpha \in \Delta} \mathrm{Cl}\left(A_{\alpha}\right)=\varnothing$, there exists a countable subfamily $\left\{\alpha_{n}: n \in\right.$ $\mathbb{N}\} \subseteq \Delta$ such that $\bigcap_{n \in \mathbb{N}} C_{\alpha_{n}}=\varnothing$.

Proof. The proof of Proposition 3.4 is similar to the proof of an analogous result for almost regular-Lindelöf spaces given by Cammaroto and Santoro (see [3, Theorem 4.10]).

\section{Weakly regular Lindelöf spaces}

DEFINITION 4.1 (see [3]). A topological space $X$ is said to be weakly regular-Lindelöf if every regular cover $\left\{U_{\alpha}: \alpha \in \Delta\right\}$ of $X$ admits a countable subset $\left\{\alpha_{n}: n \in \mathbb{N}\right\} \subseteq \Delta$ such that $X=\mathrm{Cl}\left(\bigcup_{n \in \mathbb{N}} U_{\alpha_{n}}\right)$.

It is obvious that every weakly Lindelöf space is weakly regular-Lindelöf but for the converse we introduce the following question. Is it true that every weakly regularLindelöf space is weakly Lindelöf? We can answer this question with some restrictions on the space as the following proposition shows.

Proposition 4.2. A regular and weakly regular-Lindelöf space $X$ is weakly Lindelöf.

Proof. Let $\left\{U_{\alpha}: \alpha \in \Delta\right\}$ be a regularly open cover of $X$. For each $x \in X$ there exists $\alpha_{x} \in \Delta$ such that $x \in U_{\alpha_{x}}$. Since $X$ is almost regular, then by Remark 2.3, there exists a family $\left\{W_{\alpha_{x}}: x \in X\right\}$ of regularly open sets in $X$ that forms a regular cover of the weakly regular-Lindelöf space $X$. So there exists a countable set of points 
$\left\{x_{1}, x_{2}, \ldots, x_{n}, \ldots\right\}$ of $X$ such that $X=\mathrm{Cl}\left(\cup_{n \in \mathbb{N}} W_{\alpha_{x_{n}}}\right) \subseteq \mathrm{Cl}\left(\cup_{n \in \mathbb{N}} U_{\alpha_{x_{n}}}\right)$. Since $X$ is semiregular, by [3, Proposition 3.4], $X$ is weakly Lindelöf.

COROLlary 4.3. Let $(X, \tau)$ be a regular space. Then $(X, \tau)$ is weakly Lindelöf if and only if $(X, \tau)$ is weakly regular-Lindelöf.

Proposition 4.4. If $(X, \tau)$ is regular and nearly paracompact, then $(X, \tau)$ is Lindelöf if and only if $(X, \tau)$ is weakly regular-Lindelöf.

Proof. Suppose that $(X, \tau)$ is regular, nearly paracompact and weakly regularLindelöf space. Then, by Proposition $4.2,(X, \tau)$ is weakly Lindelöf and by [3, Theorem 3.8], $(X, \tau)$ is almost Lindelöf. Since an almost Lindelöf and regular space is Lindelöf we conclude that $(X, \tau)$ is Lindelöf. The converse is obvious.

The conditions that $(X, \tau)$ is regular and nearly paracompact in Proposition 4.4 are necessary. For nearly paracompactness condition, the space $E \times E$ of Example 4.5 is regular and weakly regular-Lindelöf but it is not Lindelöf nor nearly paracompact, it is not even almost regular-Lindelöf. For the necessity of the regular condition consider the half-disc topology (see [7, Example 78]). It is known that topology is separable so weakly Lindelöf hence weakly regular-Lindelöf. Also it is neither Lindelöf nor regular. Since the semiregularization of this space is the open upper half-plane with the Euclidean topology which is paracompact, then we conclude that the half-disc topology is nearly paracompact.

It is clear that every almost regular-Lindelöf space is weakly regular-Lindelöf but the converse is not true in general, as the following example shows.

EXAMPLE 4.5. Let $E$ be the Sorgenfrey line and let $E \times E$ be its product with itself which is called the Sorgenfrey plane. It is known that $E$ is regular and separable, so $E \times E$ is regular and separable, then it is weakly Lindelöf so weakly regular-Lindelöf. Also it is known that $E \times E$ is regular but not Lindelöf, so by Corollary 2.5, $E \times E$ is not almost regular-Lindelöf, thus it is not nearly regular-Lindelöf.

The following proposition gives a characterization of weakly regular-Lindelöf spaces and its proof is similar to the proof of an analogous result for almost regular-Lindelöf spaces given by Cammaroto and Santoro (see [3, Theorem 4.10]).

Proposition 4.6. A topological space $X$ is weakly regular-Lindelöf if and only if, for every family $\left\{C_{\alpha}: \alpha \in \Delta\right\}$ of closed subsets of $X$ such that, for each $\alpha \in \Delta$, there exists an open set $A_{\alpha} \supseteq C_{\alpha}$ with $\bigcap_{\alpha \in \Delta} \mathrm{Cl}\left(A_{\alpha}\right)=\varnothing$, there exists a countable subfamily $\left\{\alpha_{n}: n \in \mathbb{N}\right\}$ such that $\operatorname{Int}\left(\bigcap_{n \in \mathbb{N}} C_{\alpha_{n}}\right)=\varnothing$.

\section{REFERENCES}

[1] G. Balasubramanian, On some generalizations of compact spaces, Glas. Mat. Ser. III 17(37) (1982), no. 2, 367-380. MR 85a:54031. Zbl 514.54015.

[2] F. Cammaroto and G. Lo Faro, Weakly compact spaces, Riv. Mat. Univ. Parma (4) 7 (1981), 383-395 (Italian). MR 83m:54040. Zbl 506.54018.

[3] F. Cammaroto and G. Santoro, Some counterexamples and properties on generalizations of Lindelöf spaces, Int. J. Math. Math. Sci. 19 (1996), no. 4, 737-746. MR 97h:54020. Zbl 860.54033 . 
[4] Z. Frolik, Generalizations of compact and Lindelöf spaces, Czechosl. Math. J. 9 (84) (1959), 172-217. Zbl 098.14201.

[5] M. K. Singal and S. P. Arya, On almost-regular spaces, Glasnik Mat. Ser. III 4 (24) (1969), 89-99. MR 39\#4804. Zbl 169.24902.

[6] _ _ On nearly paracompact spaces, Mat. Vesnik 6 (21) (1969), 3-16. MR $41 \# 4486$. Zbl 177.50802 .

[7] L. A. Steen and J. A. Seebach Jr., Counterexamples in Topology, 2nd ed., Springer-Verlag, New York, 1978. MR 80a:54001. Zbl 386.54001.

[8] S. Willard and U. N. B. Dissanayake, The almost Lindelöf degree, Canad. Math. Bull. 27 (1984), no. 4, 452-455. MR 86g:54009. Zbl 551.54003.

ANWAR JABOR FAWAKHrEH: UNIVERSITY PUTRA MALAYSia, DEPARTMENT OF MATHEMATICS, 43400 UPM, SERDANG, SELANGOR, MALAYSIA

E-mail address: abuanas7@hotmai 1.com

ADEM KILIÇMAN: UNIVERSITY PUTRA MALAYSIA, DEPARTMENT OF MATHEMATICS, 43400 UPM, SERDANG, SELANGOR, MALAYSIA

E-mail address: aki1ic@fsas.upm.edu.my 


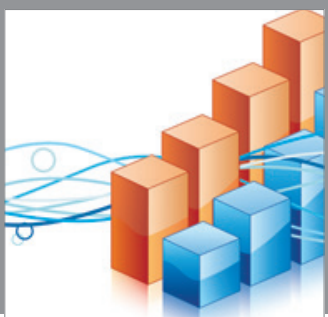

Advances in

Operations Research

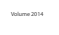

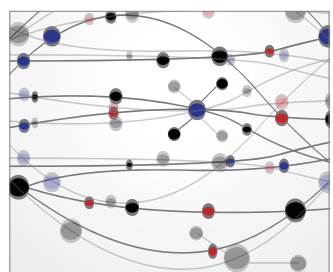

\section{The Scientific} World Journal
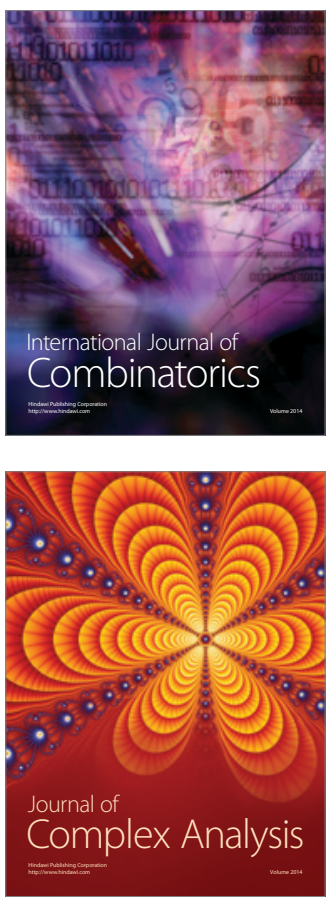

International Journal of

Mathematics and

Mathematical

Sciences
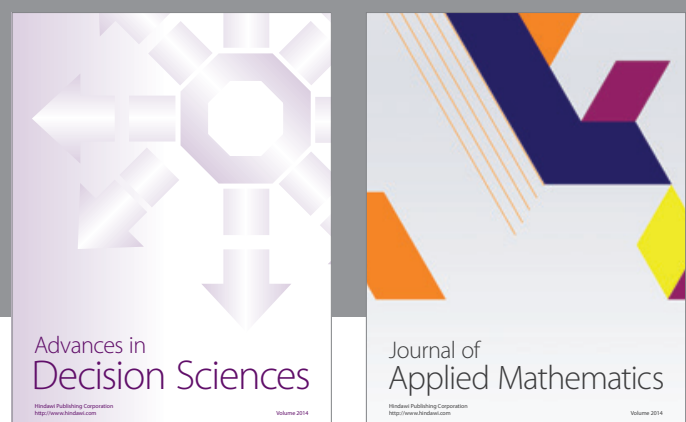

Journal of

Applied Mathematics
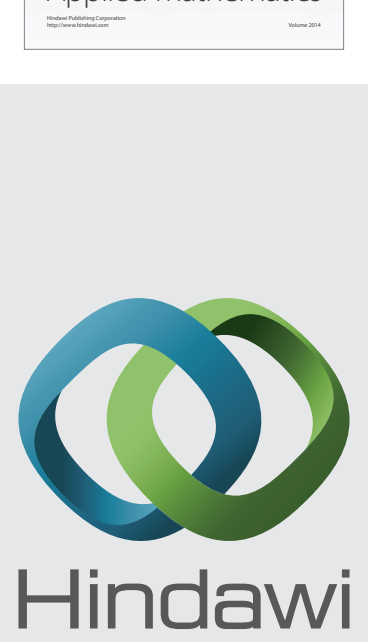

Submit your manuscripts at http://www.hindawi.com
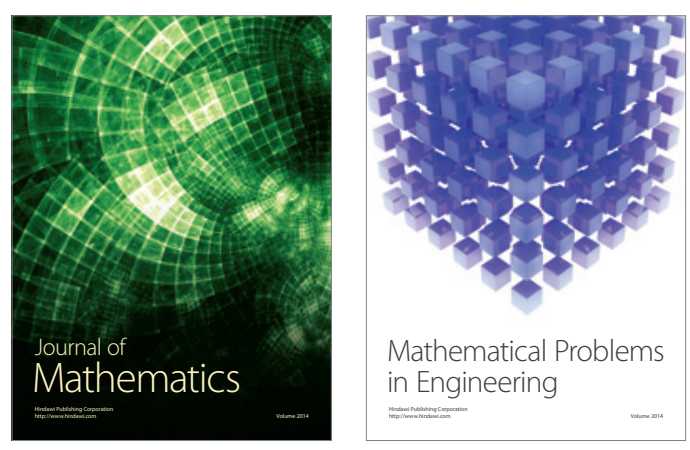

Mathematical Problems in Engineering
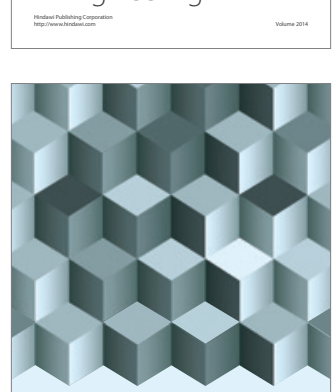

Journal of

Function Spaces
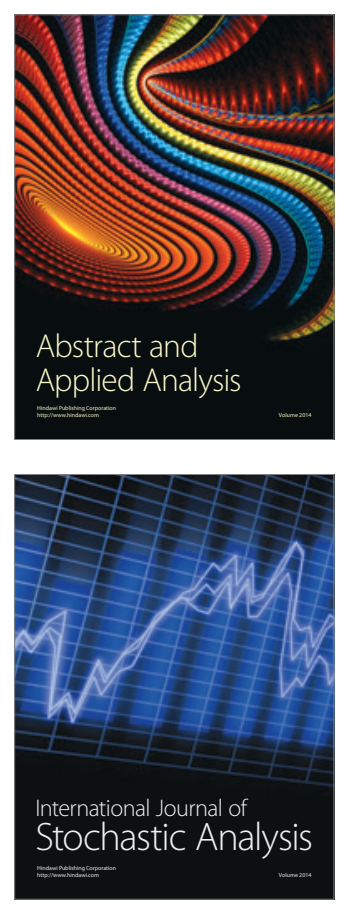

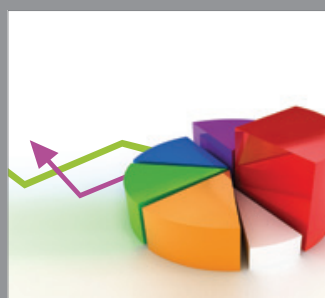

ournal of

Probability and Statistics

Promensencen
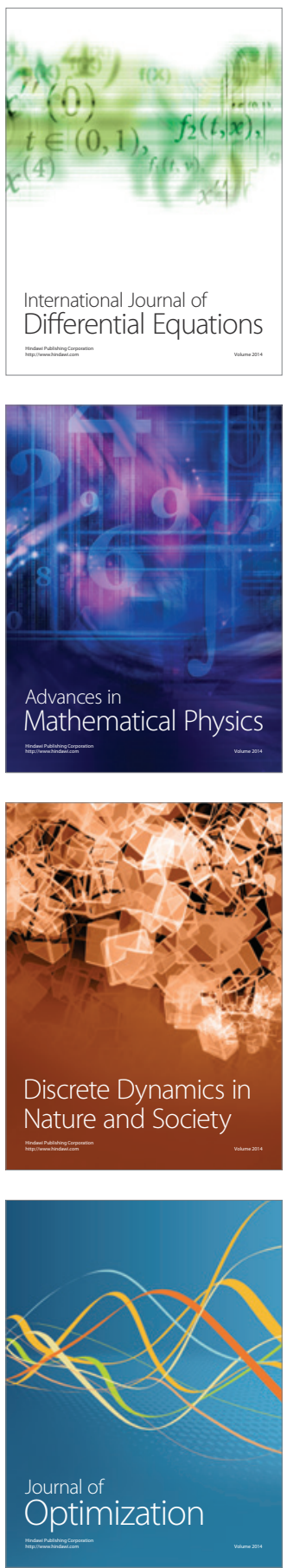